\title{
Hodge loci and absolute Hodge classes
}

\author{
Claire Voisin \\ To Joseph Steenbrink, on the occasion of his 60th birthday
}

\begin{abstract}
This paper addresses several questions related to the Hodge conjecture. First of all we consider the question, asked by Maillot and Soulé, whether the Hodge conjecture can be reduced to the case of varieties defined over number fields. We show that this is the case for the Hodge classes whose corresponding Hodge locus is defined over a number field. We also give simple criteria for this last condition to be satisfied. Finally we discuss the relation between this condition and the notion of absolute Hodge class.
\end{abstract}

\section{Introduction}

Let $\pi: \mathcal{X} \rightarrow T$ be a family of smooth projective complex varieties. Assume that $\mathcal{X}, \pi, T$ are defined over $\mathbb{Q}$. An immediate consequence of the fact that there are only countably many components of the relative Hilbert scheme for $\pi$, and that the relative Hilbert scheme (with fixed Hilbert polynomial) is defined over $\mathbb{Q}$, is the following: if the Hodge conjecture is true, the components of the Hodge locus in $T$ are defined over $\overline{\mathbb{Q}}$, and their Galois transforms are again components of the Hodge locus. (We recall later the definition of the components of the Hodge locus.) In [CDK95], it is proven that the components of the Hodge locus (and even the components of the locus of Hodge classes, which is a finer notion) are algebraic sets, while Hodge theory would give them only a local structure of closed analytic subsets (see [Voi03, § 5.3.1]).

In this paper, we give simple sufficient conditions for components of the Hodge locus to be defined over $\overline{\mathbb{Q}}$ (and their Galois transforms to also be components of the Hodge locus). Of course, this criterion does not hold in full generality and, in particular, it does not say anything about the definition field of an isolated point in the Hodge locus. However, in practice, it is reasonably easy to check and allows us to conclude in some explicit cases, where the Hodge conjecture is not known to hold. We give a few examples of applications in $\S 3$.

We first relate this geometric language to the notion of absolute Hodge classes (as we only deal with the de Rham version, we will not use the terminology of Hodge cycles of [Del82]), and explain why this notion allows us to reduce the Hodge conjecture to the case of varieties defined over $\overline{\mathbb{Q}}$, thus clarifying a question asked by Vincent Maillot and Christophe Soulé. Therefore the present work, which underlines the relation between the study of the Hodge loci and the more arithmetic notion of absolute Hodge class is related to the study of the variational form of the Hodge conjecture; see [And06].

Let us recall the notion of (de Rham) absolute Hodge class (cf. [Del82]). Let $X^{\text {an }}$ be a complex projective manifold and $\alpha \in H d g^{2 k}\left(X^{\text {an }}\right)$ be a rational Hodge class. Thus, $\alpha \in H^{2 k}\left(X^{\text {an }}, \mathbb{Q}\right)$ is

Received 30 April 2006, accepted in final form 26 November 2006.

2000 Mathematics Subject Classification 14C30, 14D07, 14F40.

Keywords: Hodge classes, Hodge loci, global invariant cycle theorem, algebraic de Rham cohomology.

This journal is (C) Foundation Compositio Mathematica 2007. 


\section{VoIsin}

rational and

$$
\alpha \in F^{k} H^{2 k}\left(X^{\text {an }}, \mathbb{C}\right) \cong \mathbb{H}^{2 k}\left(X^{\text {an }}, \Omega_{X^{\text {an }}}^{\bullet}\right) .
$$

Here, the left-hand side is Betti cohomology of the complex manifold $X^{\text {an }}$ and the isomorphism of $(0.1)$ is induced by the holomorphic Poincaré resolution

$$
0 \rightarrow \mathbb{C} \stackrel{(2 i \pi)^{k}}{\rightarrow} \mathcal{O} \stackrel{d}{\rightarrow} \Omega_{X} \rightarrow \cdots \rightarrow \Omega_{X}^{n} \rightarrow 0, \quad n=\operatorname{dim} X
$$

of the constant sheaf $\mathbb{C}$ on $X^{\text {an }}$. The right-hand side in $(0.1)$ can then be computed, by the GAGA principle, as the hypercohomology of the algebraic variety $X$ with value in the algebraic de Rham complex:

$$
\mathbb{H}^{2 k}\left(X^{\text {an }}, \Omega_{X^{\text {an }}}^{\bullet \geqslant k}\right) \cong \mathbb{H}^{2 k}\left(X, \Omega_{X / \mathbb{C}}^{\bullet \geqslant k}\right) .
$$

Let us denote by $\mathcal{E}$ the set of field embeddings of $\mathbb{C}$ in $\mathbb{C}$. For each element $\sigma$ of $\mathcal{E}$, we get a new algebraic variety $X_{\sigma}$ defined over $\mathbb{C}$, and we have a similar isomorphism for $X_{\sigma}$. Furthermore, the field embedding $\sigma$ provides a $\sigma(\mathbb{C})$-linear morphism

$$
\mathbb{H}^{2 k}\left(X, \Omega_{X / \mathbb{C}}^{\bullet \geqslant k}\right) \rightarrow \mathbb{H}^{2 k}\left(X_{\sigma}, \Omega_{X_{\sigma} / \mathbb{C}}^{\bullet \geqslant k}\right)
$$

Thus, the class $\alpha$ provides a (de Rham or Betti) complex cohomology class

$$
\alpha_{\sigma} \in \mathbb{H}^{2 k}\left(X_{\sigma}, \Omega_{X_{\sigma} / \mathbb{C}}^{\bullet \geqslant k}\right)=F^{k} H^{2 k}\left(X_{\sigma}^{\mathrm{an}}, \mathbb{C}\right)
$$

for each $\sigma \in \mathcal{E}$.

Definition 0.1 (cf. [Del82]). The class $\alpha$ is said to be (de Rham) absolute Hodge if $\alpha_{\sigma}$ is a rational cohomology class for each $\sigma$.

We introduce in $\S 1$ the notion of weakly absolute Hodge class. In the definition above, we ask that each $\alpha_{\sigma}$ is proportional to a rational cohomology class.

In this paper, we first prove the following statement, which answers a question asked by Maillot and Soulé.

Proposition 0.2. Assume that the Hodge conjecture is known for varieties $X_{\overline{\mathbb{Q}}}$ defined over $\overline{\mathbb{Q}}$ and (weakly) absolute Hodge classes $\alpha$ on them. Then the Hodge conjecture is true for (weakly) absolute Hodge classes.

Remark 0.3 . As is easy to prove, and as we will see in $\S 1$ (see Lemma 1.5), a weakly absolute Hodge class $\alpha$ on a variety $X$ defined over $\overline{\mathbb{Q}}$ is defined over $\overline{\mathbb{Q}}$, that is

$$
\alpha \in \mathbb{H}^{2 k}\left(X_{\overline{\mathbb{Q}}}, \Omega_{X_{\overline{\mathbb{Q}} / \overline{\mathbb{Q}}} \geqslant k}\right) .
$$

This is more or less contained in [Del82], and can be proved using our geometric arguments.

Remark 0.4. In the statement of the proposition, we fix an embedding of $\overline{\mathbb{Q}}$ into $\mathbb{C}$, and so $\alpha$ determines a class in $\mathbb{H}^{2 k}\left(X_{\mathbb{C}}, \Omega_{X_{\mathbb{C}} / \mathbb{C}}^{\bullet \geqslant k}\right)=F^{k} H^{2 k}\left(X_{\mathbb{C}}^{\text {an }}, \mathbb{C}\right)$, which is assumed to be rational. If the Hodge conjecture is true for this class, then for any other embedding $\sigma$ of $\overline{\mathbb{Q}}$ into $\mathbb{C}$, the class $\alpha_{\sigma}$ is also rational, and the Hodge conjecture is also true for this Hodge class. Thus, the statement makes sense and is independent of the choice of embedding.

The proof of this proposition is based on the global invariant cycles theorem of Deligne, and on a geometric reinterpretation of the notion of absolute or weakly absolute Hodge class, which is presented in $\S 1$. The proof itself also gives the following result. Let $\pi: \mathcal{X} \rightarrow T$ be a smooth projective morphism between quasi-projective varieties defined over $\mathbb{Q}$, and let $X$ be one fiber of $\pi$ over a complex point of $T$. 


\section{Hodge LOCI AND ABSOlute Hodge Classes}

Proposition 0.5. Suppose that the Hodge conjecture is true for Hodge classes on smooth projective varieties defined over $\overline{\mathbb{Q}}$. Let $\alpha$ be a Hodge class on $X$. Assume that the Hodge locus of $\alpha$ is defined over $\overline{\mathbb{Q}}$; then the class $\alpha$ is algebraic.

Here we use the following notion. The locus of Hodge classes for the family $\pi: \mathcal{X} \rightarrow T$ is the set of all Hodge classes of degree $2 k$ on some fiber of $\pi$. It is seen as a subset of the Hodge bundle $F^{k} H_{\mathbb{C}}^{2 k}$ on $T$.

The Hodge locus of $\alpha$ is defined as the projection to $T$ (via the natural map $p: F^{k} H^{2 k} \rightarrow T$ ) of the connected component of the locus of Hodge classes passing through $\alpha$.

We now turn to the problem of whether Hodge classes should be (weakly) absolute. As we will see in $\S 1$, this is exactly related to the field of definition of the connected components of the locus of the Hodge classes. In statement (2) of the theorem, we will address the weaker question of the field of definition of the corresponding Hodge locus. By Proposition 0.5, this is in fact sufficient to address the question of Maillot and Soulé.

Let $X$ be a complex projective manifold, with a deformation family $\pi: \mathcal{X} \rightarrow T$ defined over $\mathbb{Q}$ as above, and let $\alpha \in H d g^{2 k}(X)$ be a Hodge class which is primitive with respect to an ambient polarization (i.e. one coming from $\mathcal{X}$ ). If $S \subset F^{k} H^{2 k}$ is a closed analytic or algebraic subset, $\pi_{S}: \mathcal{X}_{S} \rightarrow S_{\text {red }}$ is obtained by base change $p: S_{\text {red }} \rightarrow T$. We show the following.

Theorem 0.6. We have the following.

(1) Assume that for one irreducible component $S$ passing through $\alpha$ of the locus of Hodge classes, there is no constant sub-variation of Hodge structure of $R^{2 k} \pi_{S *} \mathbb{Q}_{\text {prim }}$ on $S$, except for $\mathbb{Q} \alpha_{t}$. Then $\alpha$ is weakly absolute.

(2) Let us weaken the assumptions on $S$ by asking that any constant sub-variation of Hodge structure of $R^{2 k} \pi_{S *} \mathbb{Q}$ on $S$ is of type $(k, k)$. Then, $p\left(S_{\text {red }}\right)$ is defined over $\overline{\mathbb{Q}}$, and satisfies the property that its Galois translates are also of the form $p\left(S_{\text {red }}^{\prime}\right)$ for some irreducible component $S^{\prime}$ of the locus of Hodge classes.

Remark 0.7. We consider primitive Hodge classes in statement (1) because otherwise the assumptions are empty. We could work more generally with subvariations of Hodge structures.

Remark 0.8. Statement (1) illustrates the power of the algebraicity theorem of [CDK95], as it is an immediate application of it and of the global invariant cycles theorem.

Statement (1) will imply, by Lemma 1.5 proven in the next section, that under the same assumptions, the Hodge locus of $\alpha$ is defined over $\overline{\mathbb{Q}}$ and its image under any element of $\operatorname{Gal}(\overline{\mathbb{Q}} / \mathbb{Q})$ is again a component of the Hodge locus.

An immediate corollary of Theorem 0.6(1) is the following simple statement.

Corollary 0.9. Assume that the infinitesimal Torelli theorem holds for the variation of Hodge structure on $R^{2 k} \pi_{*} \mathbb{Q}_{\text {prim. }}$. Assume that one component $S$ passing through $\alpha$ of the locus of Hodge classes has positive dimension, and that the only proper non-trivial sub-Hodge structure of $H^{2 k}(X, \mathbb{Q})_{\text {prim }}$ is $\mathbb{Q} \alpha$. Then $\alpha$ is weakly absolute.

Note that the assumption that $S$ has positive dimension is satisfied once

$$
h^{k-1, k+1}:=\operatorname{rk} H^{k-1, k+1}(X)_{\operatorname{prim}}<\operatorname{dim} T
$$

(cf. [Voi03, Proposition 5.14]).

Proof. Indeed, a constant sub-variation of Hodge structure of $R^{2 k} \pi_{S *} \mathbb{Q}_{\text {prim }}$ on $S$ must then be (by taking the fiber at the point 0 corresponding to $X$ ) equal either to $R^{2 k} \pi_{S *} \mathbb{Q}_{\text {prim }}$ or to $\mathbb{Q} \alpha$. 


\section{VoIsin}

The first case is impossible by the Torelli assumption, and $\operatorname{dim} S>0$. Thus, the assumptions of Theorem 0.6(1) are satisfied.

Note also that statement (2) of Theorem 0.6 combined with Proposition 0.5 gives the following criterion for the Maillot-Soulé question to have a positive answer.

Corollary 0.10. Suppose that the Hodge conjecture is true for Hodge classes on smooth projective varieties defined over $\overline{\mathbb{Q}}$. Then if $\alpha$ is a Hodge class on $X$ and there exists an irreducible $S$ of the locus of Hodge classes passing through $\alpha$ satisfying the assumptions of Theorem 0.6(2), the class $\alpha$ is algebraic.

The rest of the paper is devoted to a study of the assumptions of Theorem 0.6. While the assumptions in statements (1) and (2) are of course non-trivial, it is interesting to note that they can be checked by some infinitesimal computations at the point $(X, \alpha)$, involving the infinitesimal variation of Hodge structure on the cohomology of the fibers of the family $\mathcal{X}$.

This illustrates the power of the combination of the algebraicity theorem of [CDK95], and of the global invariant cycles theorem of Deligne [Del71]: a first-order computation can lead to the conclusion that a class is absolute Hodge or that its Hodge locus is defined over $\overline{\mathbb{Q}}$.

Section 1 is devoted to the discussion of absolute and weakly absolute Hodge classes in terms of the corresponding components of the locus of Hodge classes and components of the Hodge locus.

In $\S 2$, we prove the results stated in this introduction.

In the last section, we give variants and applications of Theorem 0.6. In Theorem 3.1, we give an algebraic (Zariski open) criterion on a Hodge class $\alpha \in F^{k} H^{2 k}$ in order that the assumptions of Theorem 0.6 are satisfied at least at a general point of the connected component $\widetilde{S}_{\alpha}$ of the locus of Hodge classes passing through $\alpha$. Of course, except in level 2 where we can use the Green density criterion, it is hard to decide whether there are many Hodge classes in the Zariski open set of $F^{k} H^{2 k}$ where this criterion is satisfied. We give examples in level 2, where this criterion is satisfied in a Zariski dense open set, in which there are 'many' Hodge classes. In one of these examples, the Hodge conjecture is not known to hold for these classes.

The second application (Theorem 3.6) concerns the period map. Under a reasonable assumption on the infinitesimal variation of Hodge structures on the primitive cohomology of the fibers of a family $\pi: \mathcal{X} \rightarrow T$ of projective varieties defined over $\mathbb{Q}$, we conclude that any component $W$ dominating $T$ by the first projection of the set of pairs $\left(t, t^{\prime}\right) \in T \times T$, such that the Hodge structures on $H^{n}\left(X_{t}, \mathbb{Q}\right)_{\text {prim }}$ and $H^{n}\left(X_{t^{\prime}}, \mathbb{Q}\right)_{\text {prim }}$ are isomorphic, is defined over $\overline{\mathbb{Q}}$. This last application has some similarities with Mustafin's work [Mus85].

\section{Absolute and weakly absolute Hodge classes}

Let us introduce the following variant of the notion of absolute Hodge class.

Definition 1.1. A Hodge class $\alpha$ on a complex projective variety $X$ is said to be weakly (de Rham) absolute Hodge if for each $\sigma \in \mathcal{E}, \alpha_{\sigma}$ is a multiple $\lambda_{\sigma} \gamma_{\sigma}$, where $\gamma_{\sigma} \in H^{2 k}\left(X_{\sigma}^{\text {an }}, \mathbb{Q}\right)$ is a rational cohomology class (hence, a Hodge class) and $\lambda_{\sigma} \in \overline{\mathbb{Q}}$.

Remark 1.2. It turns out that the condition $\lambda_{\sigma} \in \overline{\mathbb{Q}}$ is automatically satisfied. In fact, one even knows that $\lambda_{\sigma}^{2} \in \mathbb{Q}_{>0}$.

Indeed, consider the primitive decomposition of $\alpha$ with respect to the polarization given by a projective embedding of $X$.

$$
\alpha=\sum_{2 k-2 r \geqslant 0,2 r \leqslant n} c_{1}(L)^{k-r} \alpha_{r}, \quad n=\operatorname{dim} X,
$$

where $\alpha_{r} \in H^{2 r}(X, \mathbb{Q})_{\text {prim }}$. 


\section{Hodge loci And ABsolute Hodge Classes}

Then the primitive decomposition of $\alpha_{\sigma}$ is given by

$$
\alpha_{\sigma}=\sum_{2 k-2 r \geqslant 0,2 r \leqslant n} c_{1}\left(L_{\sigma}\right)^{k-r} \alpha_{r, \sigma},
$$

and, thus, if $\alpha_{\sigma}=\lambda_{\sigma} \gamma_{\sigma}$, with $\gamma_{\sigma} \in H^{2 k}\left(X_{\sigma}^{\text {an }}, \mathbb{Q}\right)$, then for each $r$ we get

$$
\alpha_{r, \sigma}=\lambda_{\sigma} \gamma_{\sigma, r},
$$

where $\gamma_{\sigma, r}$ is the degree $2 r$ primitive component of $\gamma_{\sigma}$, and thus is a rational cohomology class. However, we know by the second Hodge-Riemann bilinear relations that if $\alpha_{r} \neq 0$, we have $\int_{X} c_{1}(L)^{n-2 r} \alpha_{r}^{2} \neq 0$ and, in fact, is of a sign which depends only on $r$. This is a rational number, which is also equal to $\int_{X_{\sigma}} c_{1}\left(L_{\sigma}\right)^{n-2 r} \alpha_{r, \sigma}^{2}$. On the other hand, as $\gamma_{\sigma, r}$ is a primitive rational cohomology class, we also have $\int_{X_{\sigma}} c_{1}\left(L_{\sigma}\right)^{n-2 r} \gamma_{\sigma, r}^{2} \in \mathbb{Q}$ and, thus, from the equalities

$$
\int_{X} c_{1}(L)^{n-2 r} \alpha_{r}^{2}=\int_{X_{\sigma}} c_{1}\left(L_{\sigma}\right)^{n-2 r} \alpha_{r, \sigma}^{2}=\lambda_{\sigma}^{2} \int_{X_{\sigma}} c_{1}\left(L_{\sigma}\right)^{n-2 r} \gamma_{\sigma, r}^{2},
$$

where the last term $\int_{X_{\sigma}} c_{1}\left(L_{\sigma}\right)^{n-2 r} \gamma_{\sigma, r}^{2}$ is a rational number of the same sign as $\int_{X} c_{1}(L)^{n-2 r} \alpha_{r}^{2}$, we get $\lambda_{\sigma}^{2} \in \mathbb{Q}>0$.

Geometrically, the meaning of these notions is given in Lemmas 1.4 and 1.5. It is formulated using the geometric notions of locus of Hodge classes and of Hodge loci.

Let $\pi: \mathcal{X} \rightarrow T$ be a family of deformations of $X$, which is defined over $\mathbb{Q}$ (here $T$ is not supposed to be geometrically irreducible, and thus the assumption is not restrictive on $X$ ). There is then the algebraic vector bundle $F^{k} H^{2 k}$ on $T$, defined over $\mathbb{Q}$, which is the total space of the locally free sheaf $F^{k} \mathcal{H}^{2 k}=R^{2 k} \pi_{*} \Omega_{\mathcal{X} / T}^{\bullet}$ on $T$. We will use the following terminology (see [CDK95]). The locus of Hodge classes for the family above, in degree $2 k$, is the set of pairs $\left(X_{t}, \alpha_{t}\right) \in F^{k} H^{2 k}(\mathbb{C})$ such that $\alpha_{t} \in H^{2 k}\left(X_{t}^{\text {an }}, \mathbb{C}\right)$ is rational (hence, a Hodge class).

The components of the Hodge locus are the image in $T$, via the natural projection $p: F^{k} H^{2 k} \rightarrow T$, of the connected components of the locus of Hodge classes. If $\alpha$ is a Hodge class on $X$, the Hodge locus of $\alpha$ is the image in $T$ of the connected component of the locus of Hodge classes passing through $\alpha$.

Note that the locus of Hodge classes is obviously locally a countable union of closed analytic subsets in $F^{k} H^{2 k}(\mathbb{C})$. Indeed, if $\alpha \in F^{k} H^{2 k}\left(X_{t}^{\text {an }}, \mathbb{C}\right) \cap H^{2 k}\left(X^{\text {an }}, \mathbb{Q}\right)$, then in a simply connected neighborhood $U$ of $t \in T$, we have a trivialization of the locally constant sheaf $R^{2 k} \pi_{*}^{\text {an }} \mathbb{C}$, which induces a trivialization of the corresponding holomorphic vector bundle $H_{\mathbb{C}}^{2 k}$ and gives a composed holomorphic map:

$$
\psi: F^{k} H_{\mathbb{C}}^{2 k} \hookrightarrow H_{\mathbb{C}}^{2 k} \rightarrow H^{2 k}\left(X_{t}, \mathbb{C}\right),
$$

where $H_{\mathbb{C}}^{2 k}$ is the total space of the locally free analytic sheaf

$$
\mathcal{H}^{2 k}=R^{2 k} \pi_{*} \Omega_{\mathcal{X} \text { an } / T}=R^{2 k} \pi_{*}^{\text {an }} \mathbb{C} \otimes \mathcal{O}_{T}
$$

on $T$.

Then, over $U$, the locus of Hodge classes identifies to $\psi^{-1}\left(H^{2 k}\left(X_{t}, \mathbb{Q}\right)\right)$, which is a countable union of fibers of $\psi$. As the map $\psi$ is holomorphic, this defines a natural structure of analytic subschemes on the connected components of the locus of Hodge classes.

Similarly, the local description of the Hodge locus of $\alpha$ is as follows. We can locally extend $\alpha$ to a locally constant section $\tilde{\alpha}$ of $R^{2 k} \pi_{*}^{\text {an }} \mathbb{Q}$. Then $\tilde{\alpha}$ gives, in particular, a holomorphic section of the vector bundle $\mathcal{H}^{2 k}:=R^{2 k} \pi_{*}^{\text {an }} \mathbb{C} \otimes \mathcal{O}_{T}$. Then the Hodge locus of $\alpha$ is simply defined by the condition

$$
\tilde{\alpha}^{\leqslant k-1}=0,
$$




\section{VoIsin}

where $\tilde{\alpha}^{\leqslant k-1}$ is the projection of $\tilde{\alpha}$ in the quotient $\mathcal{H}^{2 k} / F^{k} \mathcal{H}^{2 k}$. This again defines locally the analytic schematic structure of the Hodge locus of $\alpha$.

It is clear from these descriptions that the projection from the locus of Hodge classes to the Hodge locus is a local immersion which is open onto a union of local analytic branches of the Hodge locus.

Cattani, Deligne and Kaplan proved, in fact, the following much stronger result concerning the structure of the locus of Hodge classes (cf. [CDK95]).

TheOREM 1.3. The connected components of the locus of Hodge classes are algebraic subsets of the algebraic vector bundle $F^{k} H^{2 k}$.

Let us now reformulate the notion of '(weakly) absolute Hodge' using this terminology from Hodge theory.

Lemma 1.4. A Hodge class $\alpha$ on $X$ is absolute Hodge if and only if the connected component of the Hodge locus passing through $\alpha$ is defined over $\overline{\mathbb{Q}}$, and its Galois transforms under $\operatorname{Gal}(\overline{\mathbb{Q}} / \mathbb{Q})$ are again connected components of the locus of Hodge classes.

The statement that Hodge classes for the family $\pi: \mathcal{X} \rightarrow T$ are absolute is equivalent to the fact that the locus of Hodge classes is a countable union of algebraic subsets of $F^{k} H^{2 k}$ defined over $\mathbb{Q}$.

Proof. To see the first statement, we first observe that for $\sigma \in \mathcal{E}$, the transformation

$$
\alpha \in H^{2 k}(X, \mathbb{C}) \mapsto \alpha_{\sigma} \in H^{2 k}\left(X_{\sigma}, \mathbb{C}\right)
$$

is nothing but the action of $\sigma \in \mathcal{E}$ on the complex points of the algebraic variety $F^{k} H^{2 k}$ defined over $\mathbb{Q}$.

We observe now that given a variety $Y$ defined over $\mathbb{Q}$, and given a complex point $y \in Y(\mathbb{C})$, its orbit under $\mathcal{E}$ is equal to the set of complex points of $Y$ which belong to the $\mathbb{Q}$-Zariski closure $Y_{y}$ of $y$, and which do not belong to a proper algebraic subset of this closure which is defined over $\mathbb{Q}$. Inside $Y_{y}$, this is the complementary set of a countable union of Zariski closed nowhere dense subsets.

It follows from these observations that if a given Hodge class is absolute Hodge, then the complement of a countable union of nowhere dense subsets of its $\mathbb{Q}$-Zariski closure in $F^{k} H^{2 k}$ is contained in the locus of Hodge classes. On the other hand, as we mentioned above, the locus of Hodge classes is a countable union of closed algebraic subsets. This implies that any irreducible component of the $\mathbb{Q}$-Zariski closure of any point in $F^{k} H^{2 k}$ either intersects the locus of Hodge classes in a countable union of nowhere dense subsets or is contained in the locus of Hodge classes.

Thus, we conclude that a given Hodge class is absolute Hodge if and only its $\mathbb{Q}$-Zariski closure in $F^{k} H^{2 k}$ is contained in the locus of Hodge classes.

It remains to see that if the Hodge classes are absolute for our family, then the locus of Hodge classes is a countable union of closed algebraic subsets of $F^{k} H^{2 k}$ defined over $\mathbb{Q}$.

The above tells us that for any point $\alpha$ in this locus, its $\mathbb{Q}$-Zariski closure is contained in this locus. Recall now the local form of the locus of Hodge classes: it is locally a countable union of closed analytic subsets. Locally, in each branch of an analytic component, the set of points which are not $\mathbb{Q}$-Zariski dense is a countable union of proper closed analytic subsets. Choose in each branch a point $\alpha$ which does not belong to this union. Then its $\mathbb{Q}$-Zariski closure contains the whole branch.

It follows that for countably many generically chosen Hodge classes $\alpha_{i}$, the locus of Hodge classes must be equal to the union of the $\mathbb{Q}$-Zariski closures of the $\alpha_{i}$.

The statement that Hodge classes are weakly absolute Hodge implies the facts that the locus of Hodge classes is a countable union of algebraic subsets of $F^{k} H^{2 k}$ defined over $\overline{\mathbb{Q}}$ and that the 


\section{Hodge LOCI AND ABSOlute Hodge ClAsses}

Hodge locus is a countable union of algebraic subsets of $T$ defined over $\mathbb{Q}$. More precisely, we have the following.

LemmA 1.5. Let $\alpha \in H^{2 k}\left(X^{\text {an }}, \mathbb{Q}\right)$ be a weakly absolute Hodge class. Then the connected component $\widetilde{S}_{\alpha}$ of the locus of Hodge classes passing through $\alpha$ is defined (schematically) over $\overline{\mathbb{Q}}$, and so is the Hodge locus of $\alpha$. Furthermore, the Galois transforms of the Hodge locus of $\alpha$ are also (schematically) components of the Hodge locus.

Remark 1.6. As already mentioned, this says in particular that if $X$ is defined over $\overline{\mathbb{Q}}$, a weakly absolute Hodge class on $X$ is defined over $\overline{\mathbb{Q}}$, for the natural $\overline{\mathbb{Q}}$-structure of algebraic de Rham cohomology:

$$
\mathbb{H}^{2 k}\left(X, \Omega_{X / \mathbb{C}}^{\bullet} \cong \mathbb{H}^{2 k}\left(X, \Omega_{X_{\overline{\mathbb{Q}}} / \overline{\mathbb{Q}}}\right) \otimes_{\overline{\mathbb{Q}}} \mathbb{C}\right.
$$

Proof of Lemma 1.5. We know by Theorem 1.3 that $\widetilde{S}_{\alpha}$ is algebraic, and it is by definition connected. We make the base change $\widetilde{S}_{\alpha, \text { red }} \rightarrow T$, where we replace if necessary $\widetilde{S}_{\alpha}$ by a Zariski open set, in order to make the reduced scheme $\widetilde{S}_{\alpha, \text { red }}$ smooth. Then the corresponding family

$$
\pi_{\alpha}: \mathcal{X}_{\alpha} \rightarrow \widetilde{S}_{\alpha, \text { red }}
$$

admits the locally constant section $\tilde{\alpha} \in H^{0}\left(\widetilde{S}_{\alpha, \text { red }}, R^{2 k} \pi_{\alpha *} \mathbb{Q}\right)$. Indeed, $\tilde{\alpha}$ is a tautological section of $R^{2 k} \pi_{\alpha *} \Omega_{\mathcal{X}_{\alpha} / \widetilde{S}_{\alpha, \text { red }}}$, hence provides a holomorphic section of the bundle $F^{k} \mathcal{H}^{2 k}$ on $\widetilde{S}_{\alpha, \text { red }}$ with fiber $F^{k} H^{2 k}\left(X_{t}^{\text {an }}, \mathbb{C}\right)$ at $t \in \widetilde{S}_{\alpha, \text { red }}$. On the other hand, by definition of $\widetilde{S}_{\alpha, \text { red }}$, the class $\tilde{\alpha}_{t}$ is rational for any $t \in \widetilde{S}_{\alpha, \text { red }}$. Thus the section is in fact locally constant. By the global invariant cycle theorem [Del71], there exists a class $\beta \in H^{2 k}\left(\overline{\mathcal{X}}_{\alpha}, \mathbb{Q}\right)$ which is of type $(k, k)$ and restricts to $\tilde{\alpha}_{t}$ on each fiber $X_{t}$ of the family $\mathcal{X}_{\alpha}$. In fact, we can even make this class uniquely defined by choosing an ample line bundle $\mathcal{L}$ on $\overline{\mathcal{X}}_{\alpha}$, which allows us to define a polarization on $H^{2 k}\left(\overline{\mathcal{X}}_{\alpha}, \mathbb{Q}\right.$ ) (see also the proof of Proposition 0.2 or [And96] for more details). Then $\beta$ is uniquely determined if we impose that $\beta$ lies in the orthogonal complement of Ker rest ${ }_{X}$ with respect to this polarization.

Now let $\alpha$ be weakly absolute. Then, for any $\sigma \in \mathcal{E}$, the class $\beta_{\sigma}$ on $\mathcal{X}_{\alpha, \sigma}$ restricts to $\alpha_{\sigma}=\lambda_{\sigma} \gamma_{\sigma}$ on $X_{\sigma}$, where $\gamma_{\sigma}$ is rational, and is in the orthogonal complement of Ker rest $X_{\sigma}$ with respect to the polarization induced by $\mathcal{L}_{\sigma}$. It thus follows that $\left(1 / \lambda_{\sigma}\right) \beta_{\sigma}$, which restricts to $\gamma_{\sigma}$, has to be rational (hence, is a Hodge class). Let $\tilde{\gamma}$ be the locally constant section of $R^{2 k} \pi_{\alpha *} \mathbb{Q}$ on $\widetilde{S}_{\alpha, \text { red }}$ obtained by restricting $\left(1 / \lambda_{\sigma}\right) \beta_{\sigma}$. We conclude that we have an inclusion

$$
\frac{1}{\lambda_{\sigma}} \sigma\left(\widetilde{S}_{\alpha, \text { red }}\right) \subset \widetilde{S}_{\gamma_{\sigma}, \text { red }}
$$

which is easily checked to extend, in fact, to a schematic identification

$$
\frac{1}{\lambda_{\sigma}} \sigma\left(\widetilde{S}_{\alpha}\right)=\widetilde{S}_{\gamma_{\sigma}}
$$

Indeed, this follows from the flatness of the sections $\tilde{\alpha}_{\sigma}$ and $\tilde{\gamma}_{\sigma}$, from the fact that $\lambda_{\sigma}$ has to be constant along $\sigma\left(\widetilde{S}_{\alpha, \text { red }}\right)$ by formula (1.2), and from the fact that $\widetilde{S}_{\gamma_{\sigma}}$ is by definition connected.

As

$$
p\left(\frac{1}{\lambda} \sigma\left(\widetilde{S}_{\alpha}\right)\right)=p\left(\sigma\left(\widetilde{S}_{\alpha}\right)\right)=\sigma\left(p\left(\widetilde{S}_{\alpha}\right)\right),
$$

we conclude from (1.5) that the image via $\sigma$ of the Hodge locus of $\alpha$ is also a component of the Hodge locus.

Finally, to see that if $\alpha$ is weakly absolute Hodge, then $\widetilde{S}_{\alpha} \subset F^{k} H^{2 k}$ is defined over $\overline{\mathbb{Q}}$, we use equality (1.5) applied to $\sigma \in \mathcal{E}$, together with the fact noted in Remark 1.2 that $\lambda_{\sigma}^{2} \in \mathbb{Q}$. It follows that the constant $\lambda_{\sigma} \in \overline{\mathbb{Q}}$ can take only countably many values and, in particular, there are only 


\section{VoIsin}

countably many Galois transforms $\sigma\left(\widetilde{S}_{\alpha}\right)$, and as we know that $\widetilde{S}_{\alpha}$ is algebraic, this implies that $\widetilde{S}_{\alpha}$ is defined over $\overline{\mathbb{Q}}$.

\section{Proofs of Theorem 0.6 and Propositions 0.2 and 0.5}

Proof of Proposition 0.2. Let $\left(X^{\text {an }}, \alpha\right)$ be a pair consisting of a projective complex manifold and an absolute (respectively a weakly absolute) rational Hodge class. By the geometric interpretation given above, and by Lemma 1.5 in the weakly absolute case, it follows that there exist smooth irreducible quasi-projective varieties $\mathcal{X}, S$ defined over $\overline{\mathbb{Q}}$, a projective morphism $\pi: \mathcal{X} \rightarrow S$, and a locally constant global section

$$
\tilde{\alpha} \in H^{0}\left(S, R^{2 k} \pi_{*} \mathbb{Q}\right),
$$

such that $X$ is one fiber of $\pi$ and $\alpha$ is the restriction of $\tilde{\alpha}$ to this fiber. Indeed, starting from a family $\pi: \mathcal{X} \rightarrow T$ defined over $\mathbb{Q}$, we take for $S$ a desingularization of the component of the Hodge locus passing through $\alpha$, and make the base change $S \rightarrow T$.

Deligne's global invariant cycle theorem [Del71] says now that for any smooth projective compactification $\overline{\mathcal{X}}$ of $\mathcal{X}$, there exists a Hodge class $\beta \in H d g^{2 k}(\overline{\mathcal{X}})$ such that

$$
\beta_{\mid X}=\alpha .
$$

Of course, we may also choose $\overline{\mathcal{X}}$ defined over $\overline{\mathbb{Q}}$. In order to conclude, we claim that we may choose $\beta$ to be absolute Hodge (respectively, weakly absolute Hodge). Indeed, we will deduce from this, under the assumptions of Proposition 0.2, that $\beta$ is the class of an algebraic cycle and, then, so is its restriction $\alpha$.

To prove the claim, consider the morphism of rational Hodge structures

$$
H^{2 k}\left(\overline{\mathcal{X}}^{\text {an }}, \mathbb{Q}\right) \rightarrow H^{2 k}\left(X^{\text {an }}, \mathbb{Q}\right) .
$$

The left-hand side can be polarized using a ample line bundle $\mathcal{L}$ on $\overline{\mathcal{X}}$. That is, we use the Lefschetz decomposition with respect to this polarization, and change the signs of the natural intersection pairing

$$
\left(\alpha_{r}, \beta_{r}\right)=\int_{\overline{\mathcal{X}}} c_{1}(\mathcal{L})^{N-2 r} \alpha_{r} \cup \beta_{r}, \quad N=\operatorname{dim} \mathcal{X}
$$

on the pieces of the Lefschetz decomposition with $r$ even, in order to get a polarized Hodge structure. Thus, we conclude that there is an orthogonal decomposition

$$
H^{2 k}\left(\overline{\mathcal{X}}^{\mathrm{an}}, \mathbb{Q}\right)=A \oplus B
$$

into the sum of two Hodge structures, where the first is identified via restriction to its image in $H^{2 k}(X, \mathbb{Q})$ and the second is the kernel of the restriction map. Here $B$ is a sub-Hodge structure of $H^{2 k}\left(\overline{\mathcal{X}}^{\text {an }}, \mathbb{Q}\right)$ and $A$ is then defined as the orthogonal of $B$ under the metric described above on $H^{2 k}\left(\overline{\mathcal{X}}^{\mathrm{an}}, \mathbb{Q}\right)$.

We then define $\beta$ to be the unique element of $A$ which restricts to $\alpha$.

For each element $\sigma$ of $\operatorname{Gal}(\overline{\mathbb{Q}} / \mathbb{Q})$, we get a line bundle $\mathcal{L}_{\sigma}$ on $\overline{\mathcal{X}}_{\sigma}^{\text {an }}$, a sub-Hodge structure $B_{\sigma}:=$ Ker rest $_{X_{\sigma}}$, and the isomorphism

$$
H^{2 k}\left(\overline{\mathcal{X}}^{\text {an }}, \mathbb{C}\right) \cong H^{2 k}\left(\overline{\mathcal{X}}_{\sigma}^{\text {an }}, \mathbb{C}\right)
$$

commutes with restriction maps and is compatible with the polarizations given by $\mathcal{L}$ and $\mathcal{L}_{\sigma}$. Thus, we get similarly a rational sub-Hodge structure $A_{\sigma}$ of $H^{2 k}\left(\overline{\mathcal{X}}_{\sigma}^{\text {an }}, \mathbb{Q}\right)$ and there is a commutative diagram where the horizontal maps are restriction maps and thus are defined on rational cohomology, 


\section{Hodge loci AND ABSOlute Hodge Classes}

and the vertical maps are induced by the comparison isomorphism (2.1):

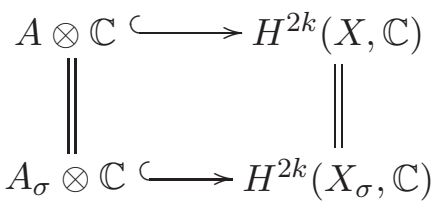

It follows from this that if $\alpha$ is absolute Hodge (respectively, weakly absolute Hodge), so is $\beta$.

Proof of Proposition 0.5. Indeed, with the same notation as above, we have the assumption that $S^{\prime}:=p\left(S_{\text {red }}\right)$ is defined over $\overline{\mathbb{Q}}$. As the monodromy acts in a finite way on the set of Hodge classes of $X_{t}, t \in S^{\prime}$ generic, there is an étale cover $S^{\prime \prime}$ of the smooth part of $S^{\prime}$, also defined over $\overline{\mathbb{Q}}$, on which this monodromy action becomes trivial. Thus, we have by base change a family $\pi^{\prime \prime}: \mathcal{X}_{S^{\prime \prime}} \rightarrow S^{\prime \prime}$, together with a global section $\tilde{\alpha}$ of $R^{2 k} \pi_{*}^{\prime \prime} \mathbb{Q}$, whose restriction to $X_{0}$ is equal to $\alpha$. The global invariant cycles theorem now says that there exists a Hodge class $\beta$ on a smooth compactification $\overline{\mathcal{X}}_{S^{\prime \prime}}$, which we may assume defined over $\overline{\mathbb{Q}}$, restricting to $\alpha$. If the Hodge conjecture is true for Hodge classes on varieties defined over $\overline{\mathbb{Q}}$, it is then true for $\beta$ and thus also for $\alpha$.

Proof of Theorem 0.6. (1) Let $(X, \alpha)$ be as in the statement of the theorem. By Theorem 1.3, the component passing through $\alpha$ of the locus of Hodge classes is an algebraic set. Let $S$ be an irreducible component of this set containing $(X, \alpha)$, and satisfying the assumption of Theorem 0.6(1). Replacing $S$ by a Zariski open set of $S_{\text {red, }}$, we may assume that $S$ is smooth. There is, by base change, a projective family $\pi_{S}: \mathcal{X}_{S} \rightarrow S$ together with a tautological flat section

$$
\tilde{\alpha} \in H^{0}\left(S, R^{2 k} \pi_{S *} \Omega_{\mathcal{X}_{S} / S}^{\bullet \geqslant k}\right),
$$

with value $\alpha_{t}$ at each $t$ (cf. the proof of Lemma 1.5).

Let $\overline{\mathcal{X}}_{S}$ be a smooth projective compactification of $\mathcal{X}_{S}$. The global invariant cycle theorem says that there exists a class $\beta \in H^{2 k}\left(\overline{\mathcal{X}}_{S}, \mathbb{Q}\right) \cap F^{k} H^{2 k}\left(\overline{\mathcal{X}}_{S}, \mathbb{Q}\right)$ such that $\beta_{\mid X}=\alpha$. On the other hand, the vector space

$$
H^{2 k}\left(\overline{\mathcal{X}}_{S}, \mathbb{Q}\right)_{\mid X_{t}} \cap H^{2 k}\left(X_{t}, \mathbb{Q}\right)_{\text {prim }}
$$

is a constant sub-Hodge structure of $H^{2 k}(X, \mathbb{Q})_{\text {prim. }}$. Thus, by our assumption on $S$, we conclude that it must be equal to $\mathbb{Q} \alpha_{t}$. It follows that the complex vector space

$$
\mathbb{H}^{2 k}\left(\overline{\mathcal{X}}_{S}, \Omega_{\overline{\mathcal{X}}_{S}}\right) \mid X_{t} \cap \mathbb{H}^{2 k}\left(X_{t}, \Omega_{X_{t}}^{\bullet}\right)_{\text {prim }}
$$

has rank 1 and is generated by $\alpha$.

Let $\sigma \in \mathcal{E}$. We want to show that the class

$$
\alpha_{\sigma} \in \mathbb{H}^{2 k}\left(X_{\sigma}, \Omega_{X_{\sigma}}^{\bullet \geqslant k}\right) \subset H^{2 k}\left(X_{\sigma}^{\text {an }}, \mathbb{C}\right)
$$

is of the form $\lambda_{\sigma} \gamma_{\sigma}$, where $\gamma_{\sigma}$ is rational.

However, $\sigma$ provides a new family $\overline{\mathcal{X}}_{S, \sigma}$ fibered over $S_{\sigma}$ with fiber $X_{t, \sigma}$, such that the vector space

$$
\mathbb{H}^{2 k}\left(\overline{\mathcal{X}}_{S, \sigma}, \Omega_{\overline{\mathcal{X}}_{S, \sigma}}\right)_{\mid X_{\sigma}} \cap \mathbb{H}^{2 k}\left(X_{\sigma}, \Omega_{X_{\sigma}}^{\bullet}\right)_{\operatorname{prim}}
$$

has rank 1 and is generated by $\alpha_{\sigma}$. It follows that the intersection of the image of the restriction map

$$
H^{2 k}\left(\overline{\mathcal{X}}_{S, \sigma}^{\mathrm{an}}, \mathbb{Q}\right) \rightarrow H^{2 k}\left(X_{\sigma}^{\mathrm{an}}, \mathbb{Q}\right),
$$

with $H^{2 k}\left(X_{\sigma}^{\text {an }}, \mathbb{Q}\right)_{\text {prim }}$ has rank 1 .

As this intersection is a sub-Hodge structure of $H^{2 k}\left(X_{\sigma}^{\text {an }}, \mathbb{Q}\right)$, we must have $\alpha_{\sigma}=\lambda_{\sigma} \gamma_{\sigma}$ for some rational primitive Hodge class $\gamma_{\sigma}$ on $X_{\sigma}$, and some non-zero complex coefficient $\lambda_{\sigma}$. By Remark 1.2, we have $\lambda_{\sigma} \in \overline{\mathbb{Q}}$, and thus $\alpha$ is weakly absolute. 


\section{VoIsin}

(2) The proof of statement (2) is similar, but needs a supplementary argument. Indeed, with the same notation as above, we find that $\alpha$ belongs to the sub-Hodge structure

$$
H^{2 k}\left(\overline{\mathcal{X}}_{S}^{\text {an }}, \mathbb{Q}\right)_{\mid X^{\text {an }}} \cap H^{2 k}\left(X^{\text {an }}, \mathbb{Q}\right)_{\text {prim }},
$$

which is the fiber at 0 of the locally constant sub-Hodge structure

$$
H^{2 k}\left(\overline{\mathcal{X}}_{S}^{\text {an }}, \mathbb{Q}\right)_{\mid X_{t}^{\text {an }}} \cap H^{2 k}\left(X_{t}^{\text {an }}, \mathbb{Q}\right)_{\text {prim }}, \quad t \in S,
$$

hence must be a trivial sub-Hodge structure. This assumption is algebraic, as it can be translated into the fact that the vector space

$$
\mathbb{H}^{2 k}\left(\overline{\mathcal{X}}_{S}, \Omega_{\overline{\mathcal{X}}_{S}}\right)_{\mid X} \cap \mathbb{H}^{2 k}\left(X, \Omega_{X}^{\bullet}\right)_{\text {prim }}
$$

is equal to

$$
\mathbb{H}^{2 k}\left(\overline{\mathcal{X}}_{S}, \Omega_{\overline{\mathcal{X}}_{S}}^{\bullet}\right)_{\mid X} \cap \mathbb{H}^{2 k}\left(X, \Omega_{X}^{\bullet \geq k}\right)_{\text {prim }} .
$$

Now let $\sigma \in \mathcal{E}$. We conclude from the above that the sub-Hodge structure

$$
H^{2 k}\left(\overline{\mathcal{X}}_{S, \sigma}^{\text {an }}, \mathbb{Q}\right)_{\mid X_{\sigma}^{\text {an }}} \cap H^{2 k}\left(X_{\sigma}^{\text {an }}, \mathbb{Q}\right)_{\text {prim }},
$$

to which $\alpha_{\sigma}$ belongs, is trivial. Thus, we can write $\alpha_{\sigma}=\sum_{i=1}^{i=N} \lambda_{i} \gamma_{i}$, where $\gamma_{i}$ are independent rational Hodge classes on $X_{\sigma}^{\text {an }}$ coming from $\overline{\mathcal{X}}_{S, \sigma}^{\text {an }}$ and the $\lambda_{i}$ are complex coefficients. As $\alpha_{\sigma}$ gives a flat section of the bundle $F^{k} \mathcal{H}^{2 k}$ on $\sigma(S)$, and the $\gamma_{i}$ are locally constant on $\sigma\left(S_{\text {red }}\right)$, we conclude that the $\lambda_{i}$ are constant on $\sigma\left(S_{\text {red }}\right)$. We claim that for a generic choice of rational coefficients $\lambda_{i}^{\prime}, 1 \leqslant i \leqslant N, \sigma\left(p\left(S_{\text {red }}\right)\right)$ is equal to $p\left(S_{\text {red }}^{\prime \prime}\right)$ where $S^{\prime \prime}$ is an irreducible component of the locus of Hodge classes passing through $\sum_{i=1}^{i=N} \lambda_{i}^{\prime} \gamma_{i}$.

Assuming the claim, this shows that there are only countably many transforms of $p\left(S_{\text {red }}\right)$ under the action of $\mathcal{E}$ and, thus, because $p\left(S_{\text {red }}\right)$ is algebraic, this implies that $p\left(S_{\text {red }}\right)$ is defined over $\overline{\mathbb{Q}}$. The claim also gives the second part of the statement.

To prove the claim, we choose a simply connected neighborhood $U$ of the point $\sigma(0) \in T(\mathbb{C})$. Over $U$, we can consider the map $\psi: F^{k} H_{\mathbb{C} \mid U}^{2 k} \rightarrow H^{2 k}\left(X_{\sigma}^{\text {an }}, \mathbb{C}\right)$ of $(1.3)$. Then for any choice of complex coefficients $\mu_{i}$, we know that $p\left(\psi^{-1}\left(\sum_{i} \mu_{i} \gamma_{i}\right)\right)$ contains $p\left(\sigma\left(S_{\text {red }}\right)\right) \cap U$, and that for $\left(\mu_{1}, \ldots, \mu_{N}\right)=$ $\left(\lambda_{1}, \ldots, \lambda_{N}\right), p\left(\sigma\left(S_{\text {red }}\right)\right) \cap U$ is the reduction of an irreducible component of $p\left(\psi^{-1}\left(\sum_{i} \mu_{i} \gamma_{i}\right)\right)$. By lower semi-continuity of the dimension of the fibers of $\psi$, we conclude that the latter property remains true for $\left(\mu_{i}\right) \in \mathbb{C}^{N}$ in a Zariski open set of coefficients and, thus, in particular for some $N$-uple $\left(\lambda_{i}^{\prime}\right) \in \mathbb{Q}^{N}$.

Having this, we proved that for some irreducible analytic component $S^{\prime}$ of $\psi^{-1}\left(\sum_{i=1}^{i=N} \lambda_{i}^{\prime} \gamma_{i}\right)$, the two analytic subsets $\sigma\left(p\left(S_{\text {red }}\right)\right) \cap U$ and $p\left(S_{\text {red }}^{\prime}\right)$ of $U$ coincide. As $\sigma\left(p\left(S_{\text {red }}\right)\right)$ is irreducible and reduced and because, by Theorem 1.3, $\psi^{-1}\left(\sum_{i=1}^{i=N} \lambda_{i}^{\prime} \gamma_{i}\right)$ is an open set in an irreducible algebraic subset $S^{\prime \prime}$ of $F^{k} H^{2 k}$ (an irreducible component of a connected component of the locus of Hodge classes), we obtain by analytic continuation that $\sigma\left(p\left(S_{\text {red }}\right)\right)=p\left(S_{\text {red }}^{\prime \prime}\right)$.

Remark 2.1. The schematic structure of the locus where a combination $\sum_{i} \mu_{i} \gamma_{i}$ remains in $F^{k} H^{2 k}$ may depend on the $\mu_{i}$, even if we know that the corresponding reduced algebraic set does not depend generically on the $\mu_{i}$. This is why we have to restrict here to the underlying reduced subschemes.

\section{Variants and applications}

Let us give to start with an infinitesimal criterion which will guarantee that the assumptions of Theorem 0.6(1) are satisfied by an irreducible component of $\widetilde{S}_{\alpha}$. This will then give as a consequence of Theorem 0.6 an algebraic criterion (Theorem 3.1) for a Hodge class $\alpha \in F^{k} H^{2 k}(\mathbb{C})$, to be weakly absolute. 


\section{Hodge LOCI AND ABSOlute Hodge Classes}

We assume again that $\pi: \mathcal{X} \rightarrow T$ is a family of projective varieties defined over $\mathbb{Q}$, and we denote as before by $F^{k} H^{2 k}$ the algebraic vector bundle whose sheaf of sections is $\left(R^{2 k} \pi_{*} \Omega_{\mathcal{X} / T}^{\bullet \geqslant k}\right)_{\text {prim }}$, which admits as a quotient the bundle $H^{k, k}$ whose sheaf of sections is $\left(R^{k} \pi_{*} \Omega_{\mathcal{X} / T}^{k}\right)_{\text {prim }}$. This is an algebraic vector bundle defined over $\mathbb{Q}$. We have the $\mathcal{O}_{T}$-linear map which describes the infinitesimal variation of Hodge structure

$$
\bar{\nabla}: \mathcal{H}^{p, q} \rightarrow \mathcal{H}^{p-1, q+1} \otimes \Omega_{T},
$$

which is defined using the Gauss-Manin connection and Griffiths transversality (cf. [Voi03, § 5.1.2]). Here $\mathcal{H}^{p, q}:=\left(R^{q} \pi_{*} \Omega_{\mathcal{X} / T}^{p}\right)_{\text {prim }}$.

The assumption of positive dimension for the Hodge loci is automatically satisfied if $h^{k-1, k+1}$ $(X)_{\text {prim }}<\operatorname{dim} T$. This is proved in [Voi03], where it is shown that the Hodge loci in $T$ for the variation of Hodge structure on $H^{k-1, k+1}\left(X_{t}\right)_{\text {prim }}$ can be defined by at most $h^{k-1, k+1}(X)_{\text {prim }}<$ $\operatorname{dim} T$ equations. We assume below that $T$ is smooth.

Let $\alpha \in H^{2 k}(X, \mathbb{Q})_{\text {prim }}$ be a Hodge class, where $X=X_{0}$ is a fiber of $\pi, 0 \in T(\mathbb{C})$. Let $\lambda \in H^{k, k}$ be the projection of $\alpha \in F^{k} H^{2 k}$ in $H^{k, k}$.

Let us assume that the map

$$
\mu_{\lambda}: T_{T, 0} \rightarrow H^{k-1, k+1}\left(X_{0}\right)
$$

given by $\mu_{\lambda}(v)=\bar{\nabla}_{v}(\lambda)$ is surjective. Let $K_{\lambda}$ be its kernel; $K_{\lambda}$ is the tangent space of the Hodge locus of $\alpha$ at 0 (cf. [Voi03, 5.3.2]). We have the following algebraic criterion on $\lambda$, for $\alpha$ to be weakly absolute.

Theorem 3.1. Assume that:

(1) $\mu_{\lambda}$ is surjective;

(2) for $p>k, p+q=2 k$, the map

$$
\bar{\nabla}_{0}: H^{p, q}\left(X_{0}\right)_{\text {prim }} \rightarrow H^{p-1, q+1}\left(X_{0}\right) \otimes K_{\lambda}^{*},
$$

obtained by restriction of $\bar{\nabla}$, is injective;

(3) the map

$$
H^{k, k}\left(X_{0}\right)_{\text {prim }} \rightarrow H^{k-1, k+1}\left(X_{0}\right) \otimes K_{\lambda}^{*},
$$

obtained by restriction of $\bar{\nabla}$, has for its kernel the line generated by $\lambda$.

Then $\alpha$ is weakly absolute.

Proof. As the map $\mu_{\lambda}$ is surjective, the component $S_{\alpha}$ of the Hodge locus determined by $\alpha$ is smooth with tangent space $K_{\lambda}$ at $0 \in T$ (cf. [Voi03, Proposition 5.14]).

Conditions (2) and (3) imply that any constant sub-variation of Hodge structure of $R^{2 k} \pi_{*} \mathbb{Q}_{\text {prim }}$ defined along an open set of $S_{\alpha}$ containing the point 0 parameterizing $X$ is equal to $\mathbb{Q} \alpha$. Indeed, if $\gamma^{p, q}$ is a locally constant section of $R^{2 k} \pi_{*} \mathbb{C}_{\text {prim }}$ which remains of type $(p, q)$ near 0 on $S_{\alpha}$, where we may assume $p \geqslant q$ by Hodge symmetry, then we have

$$
\bar{\nabla} \gamma^{p, q}=0 \quad \text { in } \mathcal{H}^{p-1, q+1} \otimes \Omega_{S_{\alpha}},
$$

and, in particular, we have (at 0 )

$$
\bar{\nabla} \gamma^{p, q}(0)=0 \quad \text { in } H^{p-1, q+1}\left(X_{0}\right)_{\operatorname{prim}} \otimes K_{\lambda}^{*} .
$$

Thus, by assumptions (2) and (3), we conclude that $\gamma^{p, q}=0$ for $p>k$ and $\gamma^{p, q}$ is proportional to $\lambda$ for $p=k$.

We conclude then by applying Theorem 0.6(1).

Remark 3.2. The same reasoning shows that if we only assume that conditions (1) and (2) in Theorem 3.1 hold, then the class $\alpha$ satisfies the conclusion of part (2) of Theorem 0.6. Thus, 


\section{VoIsin}

in particular, if $\widetilde{S}_{\alpha}^{0}$ is the irreducible component of $\widetilde{S}_{\alpha}$ passing through $\alpha$ (it is unique and reduced because $\widetilde{S}_{\alpha}$ is now smooth at the point $\left.\alpha\right)$, then $p\left(\widetilde{S}_{\alpha}^{0}\right)$ is defined over $\overline{\mathbb{Q}}$.

It is interesting to note that condition (1) is a Zariski open condition on the class $\lambda \in H^{k, k}$ (not necessarily Hodge) and that conditions (2) and (3) are Zariski open in the set where condition (1) is satisfied. One can even note that the complementary set where these conditions are not satisfied is Zariski closed and defined over $\mathbb{Q}$, as are the bundles $\mathcal{H}^{p, q}$ and the map $\bar{\nabla}$.

Of course, even if we can show that the Zariski open set of $F^{k} H^{2 k}$ defined by conditions (1)-(3) above is non-empty, it is not clear whether there are any Hodge classes in it. This is the case, however, if our variation of Hodge structure has Hodge level 2 , that is, $h^{p, q}=0$ for $p \geqslant k+2$. Indeed, in this case, we have the Green density criterion given below which guarantees that if there is any $\lambda \in H^{k, k}\left(X_{t}\right)_{\text {prim }}$ satisfying property (1), then the set of rational Hodge classes is topologically dense in the real part of the vector bundle $H^{k, k}$. The general statement is as follows. Let $H$ be a local system of rational vector spaces on a smooth complex connected basis $B$, and suppose that there is a variation of Hodge structure of weight 2 on $H$, that is, a holomorphically varying Hodge filtration

$$
\mathcal{H}^{2,0} \subset F^{1} \mathcal{H} \subset F^{0} \mathcal{H}=\mathcal{H}:=H \otimes_{\mathbb{Q}} \mathcal{O}_{B}
$$

satisfying the condition that

$$
F^{1} \mathcal{H} \oplus \overline{\mathcal{H}^{2,0}}=\mathcal{H}
$$

and Griffiths transversality

$$
\nabla\left(F^{i} \mathcal{H}\right) \subset F^{i-1} \mathcal{H} \otimes \Omega_{B} .
$$

Let $\mathcal{H}^{1,1}:=F^{1} \mathcal{H} / F^{2} \mathcal{H} ; \mathcal{H}^{1,1}$ also identifies to the $C^{\infty}$-subbundle of $H \otimes \mathcal{C}^{\infty}$ with fiber

$$
F^{1} H_{b} \cap \overline{F^{1} H_{b}}
$$

over $b \in B$. Furthermore, it has a real structure

$$
\mathcal{H}^{1,1}=\mathcal{H}_{\mathbb{R}}^{1,1} \otimes \mathbb{C},
$$

where $\mathcal{H}_{\mathbb{R}}^{1,1}$ is the real vector bundle with fiber $H_{\mathbb{R}} \cap F^{1} \mathcal{H}_{b}$ over $b \in B$.

The infinitesimal variation of Hodge structure gives an $\mathcal{O}_{B}$-linear map

$$
\bar{\nabla}: T_{B} \rightarrow \operatorname{Hom}\left(\mathcal{H}^{1,1}, \mathcal{H}^{0,2}\right)
$$

and, thus, for any $\lambda \in H^{1,1}\left(X_{b}\right)$, a $\mathbb{C}$-linear map on the fibers

$$
\mu_{\lambda}: T_{B, b} \rightarrow \mathcal{H}_{b}^{0,2}, \quad \mu_{\lambda}(u)=\bar{\nabla}_{u}(\lambda) .
$$

Proposition 3.3 (cf. [Voi03, §5.3.4]). Assume that for one $b \in B$, there exists a $\lambda \in H_{b}^{1,1}$ such that $\mu_{\lambda}$ is surjective. Then the locus of Hodge classes is topologically dense in the real vector bundle $\mathcal{H}_{\mathbb{R}}^{1,1}$.

Example 3.4. The criterion above allows us to prove that many Hodge classes are weakly absolute for surfaces in $\mathbb{P}^{3}$, without using the Lefschetz theorem on $(1,1)$-classes.

More interestingly, it allows us to show a similar result for certain level 2 subvariations of Hodge structure in the $H^{2 k}$ of a variety $X$, without knowing the Hodge conjecture for the Hodge classes in this sub-Hodge structure. We can construct such examples on four-dimensional hypersurfaces with automorphisms.

Example 3.5. Consider the action of the involution $\iota$ on $\mathbb{P}^{5}$ given by $\iota\left(X_{0}, \ldots, X_{5}\right)=\left(-X_{0},-X_{1}\right.$, $\left.X_{2}, \ldots, X_{5}\right)$, and take for $T$ the family of isomorphism classes of degree 6 hypersurfaces whose defining equation is invariant under $\iota$, and for sub-Hodge structure the anti-invariant part of $H^{4}(X)$ under $\iota$. This Hodge structure has Hodge level 2, because $\iota$ acts trivially on the rank 1 space $H^{4,0}(X)$. 


\section{Hodge loci And ABsolute Hodge Classes}

Thus, the Green density criterion applies once assumption (1) above is satisfied. The parameter space $T$ has dimension 226, and the number $h_{-}^{1,3}$ is equal to 208. One can check that assumptions (1)-(3) are satisfied generically on $F^{2} H_{-}^{4}$, thus proving that many Hodge classes are weakly absolute, even if the Hodge conjecture is not known for them. This is done following [CG80, Gri69] by computations in the Jacobian ring of the generic hypersurface as above. In fact, this can be done for $X$ the Fermat hypersurface, and for a generic class $\lambda \in H^{2,2}(X)_{-}$.

We now turn to another application of Theorem 0.6, which concerns the fibers of the period map and the Torelli problem.

Let $\pi: \mathcal{X} \rightarrow T$ be a family of smooth polarized projective varieties which is defined over $\mathbb{Q}$, and consider the variation of Hodge structure on $H^{n}\left(X_{t}\right)_{\text {prim }}$. Here $T$ is assumed to be smooth. The corresponding infinitesimal variation of Hodge structure at $t \in T$ is given by the map

$$
\bar{\nabla}: H^{p, q}\left(X_{t}\right)_{\text {prim }} \rightarrow H^{p-1, q+1}\left(X_{t}\right)_{\text {prim }} \otimes \Omega_{T, t} .
$$

We assume the following property: at the generic point $0 \in T$, the corresponding map

$$
\begin{aligned}
\mu: H^{p, q}\left(X_{0}\right)_{\operatorname{prim}} \otimes T_{T, 0} & \rightarrow H^{p-1, q+1}\left(X_{0}\right)_{\text {prim }}, \\
\eta \otimes v & \mapsto \bar{\nabla}_{v}(\eta),
\end{aligned}
$$

is surjective whenever $H^{p, q}\left(X_{0}\right)_{\text {prim }} \neq 0$. (This property is satisfied, for example, by the families of hypersurfaces or complete intersections in projective space.) We then have the following.

TheOREM 3.6. Let $Z \subset T \times T$ be the set of points $\left(t, t^{\prime}\right)$ such that there exists an isomorphism of Hodge structures between $H^{n}\left(X_{t}, \mathbb{Q}\right)_{\text {prim }}$ and $H^{n}\left(X_{t^{\prime}}, \mathbb{Q}\right)_{\text {prim }}$. Let $W \subset T \times T$ be (the underlying reduced scheme of) an irreducible component of $Z$ which dominates $T$. Then under the assumptions above, $W$ is defined over $\overline{\mathbb{Q}}$ and any Galois transform of $W$ is again (the underlying reduced scheme of) an irreducible component of $Z$.

Proof. We apply Theorem $0.6(2)$. The set $W$ above is $\Gamma_{\text {red }}$ for an irreducible component $\Gamma$ of the Hodge locus corresponding to the induced variation of Hodge structure of weight 0 on $H^{n}$ $\left(X_{t}, \mathbb{Q}\right)_{\text {prim }}^{*} \otimes H^{n}\left(X_{t^{\prime}}, \mathbb{Q}\right)_{\text {prim }}$ on $T \times T$. What we have to prove in order to apply Theorem 0.6(2) is the fact that if $W$ dominates $T$ by the first (or equivalently second) projection, then any constant sub-Hodge structure of

$$
H^{n}\left(X_{t}, \mathbb{Q}\right)_{\operatorname{prim}}^{*} \otimes H^{n}\left(X_{t^{\prime}}, \mathbb{Q}\right)_{\text {prim }}, \quad\left(t, t^{\prime}\right) \in W
$$

must be of type $(0,0)$.

By definition, for $\left(t, t^{\prime}\right) \in W$, the Hodge structures on

$$
H^{n}\left(X_{t}, \mathbb{Q}\right)_{\text {prim }}, \quad H^{n}\left(X_{t^{\prime}}, \mathbb{Q}\right)_{\text {prim }},
$$

are isomorphic. Thus, the Hodge structures on

$$
H^{n}\left(X_{t}, \mathbb{Q}\right)_{\text {prim }}^{*} \otimes H^{n}\left(X_{t^{\prime}}, \mathbb{Q}\right)_{\text {prim }}, \quad H^{n}\left(X_{t}, \mathbb{Q}\right)_{\text {prim }}^{*} \otimes H^{n}\left(X_{t}, \mathbb{Q}\right)_{\text {prim }}
$$

are isomorphic. Furthermore, $t$ is generic. Thus, it suffices to prove that on any finite cover of $T$, there is no constant sub-Hodge structure of $H^{n}\left(X_{t}, \mathbb{Q}\right)_{\operatorname{prim}}^{*} \otimes H^{n}\left(X_{t}, \mathbb{Q}\right)_{\text {prim }}$ which is not of type $(0,0)$.

This is done by an easy infinitesimal argument. Let

$$
\alpha \in H^{n}\left(X_{t}, \mathbb{Q}\right)_{\text {prim }}^{*} \otimes H^{n}\left(X_{t}, \mathbb{Q}\right)_{\text {prim }}
$$

be of bidegree $(r, s)$ with $r>s, r+s=0$. Thus, $r>0$ and if we see $\alpha$ as an element of $\operatorname{Hom}\left(H^{n}\left(X_{t}\right)_{\text {prim }}, H^{n}\left(X_{t}\right)_{\text {prim }}\right), \alpha \in H^{r,-r}$ means that $\alpha\left(H^{p, q}\left(X_{t}\right)_{\text {prim }}\right) \subset H^{p+r, q-r}\left(X_{t}\right)_{\text {prim }}$.

We have to show that if there is a flat section $\tilde{\alpha}$ on $T$, extending $\alpha$ and staying of type $(r,-r)$, with $r>0$, then $\alpha=0$. It suffices to show this at first order at $0 \in T$, where this is equivalent to 


\section{Hodge LOCI AND ABSOlute Hodge ClAsses}

saying that if

$$
\bar{\nabla} \alpha=0 \quad \text { in } H^{r-1,-r+1}\left(X_{0} \times X_{0}\right) \otimes \Omega_{T, 0},
$$

then $\alpha=0$. However, saying that $\bar{\nabla} \alpha=0$ is equivalent to saying that

$$
\bar{\nabla}_{v}(\alpha(\phi))=\alpha\left(\bar{\nabla}_{v}(\phi)\right), \quad \forall \phi \in H^{p, q}\left(X_{0}\right)_{\text {prim }}, \forall(p, q), p+q=n, \forall v \in T_{T, 0} .
$$

Equation (3.1) shows that $\alpha$ is, in fact, determined by its value on the first non-zero term $H^{p, q}$ $\left(X_{0}\right)_{\text {prim }}$, because, by assumption, the map

$$
\begin{aligned}
H^{p, q}\left(X_{0}\right)_{\text {prim }} \otimes T_{T, 0} & \rightarrow H^{p-1, q+1}\left(X_{0}\right)_{\text {prim }}, \\
\phi \otimes v & \mapsto \bar{\nabla}_{v}(\phi)
\end{aligned}
$$

is surjective once $H^{p, q}\left(X_{0}\right)_{\text {prim }}$ is different from zero.

On the other hand, $\alpha$ must be zero on the first non-zero term $H^{p, q}\left(X_{0}\right)_{\text {prim }}$, because it sends $H^{p, q}\left(X_{0}\right)_{\text {prim }}$ in $H^{p+r, q-r}\left(X_{0}\right)_{\text {prim }}$, which is zero because $r>0$.

\section{ACKNowledgements}

I thank Vincent Maillot and Christophe Soulé for asking the question addressed in this paper, whether the Hodge conjecture could be reduced to the case of varieties defined over $\overline{\mathbb{Q}}$. I am also grateful to Yves André and the referee for helpful comments and suggestions.

It is my great pleasure to dedicate this paper to Joseph Steenbrink, who contributed in a major way to Hodge theory.

\section{REFERENCES}

And96 Y. André, Pour une théorie inconditionnelle des motifs, Publ. Math. Inst. Hautes Études Sci. 83 (1996), 5-49.

And06 Y. André, Déformation et spécialisation de cycles motivés, J. Inst. Math. Jussieu 5 (2006), 563-603.

CG80 J. Carlson and Ph. Griffiths, Infinitesimal variation of Hodge structure and the global Torelli problem, in Géométrie algébrique, Angers, 1980 (ed. A. Beauville; Sijthoff-Noordhoff, Alphen aan den Rijn, 1980), 51-76.

CDK95 E. Cattani, P. Deligne and A. Kaplan, On the locus of Hodge classes, J. Amer. Math. Soc. 8 (1995), 483-506.

Del71 P. Deligne, Théorie de Hodge II, Publ. Math. Inst. Hautes Études Sci. 40 (1971), 5-57.

Del82 P. Deligne, Hodge cycles on abelian varieties (notes by J. S. Milne), Lecture Notes in Mathematics, vol. 900 (Springer, Berlin, 1982), 9-100.

Gri69 Ph. Griffiths, On the periods of certain rational integrals I, II, Ann. of Math. (2) 90 (1969), $460-541$.

Mus85 G. A. Mustafin, Families of algebraic varieties and invariant cycles, Izv. Akad. Nauk SSSR Ser. Mat. 49 (1985), 948-978, 1119.

Voi03 C. Voisin, Hodge theory and complex algebraic geometry II, Cambridge Studies in Advanced Mathematics, vol. 77 (Cambridge University Press, Cambridge, 2003).

Claire Voisin voisin@math.jussieu.fr

Institut de Mathématiques de Jussieu, CNRS, UMR 7586, 175, rue du Chevaleret, 75013 Paris, France 